\title{
Occult macular dystrophy in an Italian family carrying a mutation in the RP1L1 gene
}

\author{
STEFANO PIERMAROCCHI $^{1}$, TATIANA SEGATO ${ }^{1}$, ALBERTA LEON $^{2}$, \\ DAVIDE COLAVITO $^{2}$ and STEFANIA MIOTTO ${ }^{1}$ \\ ${ }^{1}$ Department of Neuroscience, University of Padova, Padova IT-35128; \\ ${ }^{2}$ Research and Innovation Laboratories Srl, Padova IT-35127, Italy
}

Received March 19, 2015; Accepted December 2, 2015

DOI: $10.3892 / \mathrm{mmr} .2016 .4784$

\begin{abstract}
Occult macular dystrophy (OMD) is an inherited macular disease characterized by progressive visual decline with the absence of visible retinal abnormalities. Typical alterations of the retinal structure are detectable by spectral domain optical coherence tomography (SD-OCT). Mutations in the RP1L1 gene have been identified to be responsible for the disease in Asian subjects. The present study assessed the role of mutations in the RP1L1 gene in an Italian family with OMD. One patient with OMD and five related subjects (two male offspring affected by progressive visual decline and three asymptomatic siblings of the patient) were subjected to complete ophthalmological examination. SD-OCT was also performed. All subjects were screened for OMD-associated genetic mutations in the RP1L1 gene. The OMD patient and the two symptomatic offspring presented with a reduced best-corrected visual acuity. Although no fundus abnormalities were observed, SD-OCT examination showed that the external limiting membrane and the inner segment/outer segment band were not clearly identifiable and a focal disruption of the photoreceptor layer was present. The degree of photoreceptor alterations was correlated with the severity of visual impairment. Clinical and tomographic results in the three asymptomatic relatives were normal. A p.Arg45Trp mutation in the RP1L1 gene was identified in the OMD patient, in the two symptomatic offspring and also in two of the asymptomatic siblings of the patient. The identification of RP1L1 mutations in subjects with OMD may improve the accuracy of diagnosis of this rare condition and may aid in enhancing the efficacy of genetic counseling.
\end{abstract}

Correspondence to: Dr Davide Colavito, Research and Innovation Laboratories Srl, Corso Stati Uniti 4 int F, Padova IT-35127, Italy

E-mail: colavito@researchinnovation.com

Key words: RP1L1, occult macular distrophy, progressive visual decline, macula, genetic testing

\section{Introduction}

Occult macular dystrophy (OMD) is an inherited macular disease characterized by progressive visual decline with the absence of visible fundus abnormalities (1). The condition was first recognized in 1989 as an autosomal dominant trait, although sporadic cases have also been reported (1-3). In patients with OMD, the full-field electroretinogram (ERG) is normal; however, the focal macular ERG and multifocal ERG (mfERG) of the macular area are affected, revealing localized dysfunction of the photoreceptors $(1,4,5)$.

Despite normal fundus appearance, spectral domain optical coherence tomography (SD-OCT) reveals various degrees of macular changes in OMD patients. Typical OCT features of OMD patients are a disruption of the inner segment/outer segment (IS/OS) junction and the disappearance of the cone outer segment tip (COST) line (6). Subtle changes of normal macular reflectance may also be noted in infrared reflectance images of the fundus (7).

Mutations in the retinitis pigmentosa 1-like 1 (RP1L1) gene have been identified in four Japanese families with OMD (8). To date, the association between OMD and RP1L1 mutations has mostly been reported in Asian subjects (7-10). Only two studies on Caucasian subjects with OMD are available, of which only one included an analysis for RP1L1 gene mutations $(11,12)$. The present study was the first to report on an Italian family with typical OMD and a RP1L1 mutation.

\section{Materials and methods}

Patient examination and clinical data collection. In 2013, a Caucasian family comprising several members diagnosed with OMD was assessed. In accordance with the Declaration of Helsinki, all patients provided written informed consent to full ophthalmological examination and genetic testing for OMD mutations. All subjects were evaluated by expert ophthalmologists at the Opthalmology Associates in Padova (Italy). Each subject underwent complete ophthalmological assessment, including best-corrected visual acuity (BCVA), slit-lamp examination, IOP measurement and ophthalmoscopy. Color vision testing was performed using Ishihara pseudochromatic plates. High-resolution macular scans were obtained by SD-OCT (3D-OCT 2000; Topcon, GB Ltd., 
Table I. Primer sequences.

\begin{tabular}{llc}
\hline Primer name & \multicolumn{1}{c}{ Primer sequence } & Mutations \\
\hline RP1L1 1199 Fw & GGGCTCTCATAAGTTCTTGAATCAG & Ser1199Cys \\
RP1L1 1199 Rv & GCAGATCATGAGGGCGCT & Trp960Arg \\
RP1L1 960 Fw & AAGGAGAAGCAGTGCCAGCC \\
RP1L1 960 Rv & TGCTGTCCCGCCTGAGC & Arg45Trp \\
RP1L1 45 Fw & AAGAGACAGGAAATGCCAATCC \\
RP1L1 45 Rv & TCTTATCAGAGCAGAGGTAGCAGC \\
RP1L1 2311 Fw & CTTCACTGGCCCCCTGCT & Gln2311Pro, Gln2311Glu \\
RP1L1 2311 Rv & GCCCTCAGGTCAGTCTAGGAGAT & Asp1425Glu, Asp1425His \\
RP1L1 1425 Fw & CGTGTGCTCTTGGCCCAT & \\
RP1L1 1425 Rv & TGCAGTTAGAGGAAGTTAAAGAAGGG & \\
\hline
\end{tabular}

FW, forward; Rv, reverse.

Newbury, UK). The medical records of the subjects were also thoroughly analyzed.

Genetic testing. Genetic testing was performed at the Research and Innovation Laboratories Srl (Padova, Italy). DNA extraction was performed from peripheral blood using the Qiagen Biorobot blood extraction kit (Qiagen, Hilden, Germany) according to maufacturer's instructions. $100 \mathrm{ng}$ DNA were amplified by standard polymerase chain reaction (PCR) procedures with the PCR mixture containing $2.5 \mu \mathrm{l} 10 \mathrm{X}$ concentrated PCR buffer (Solis Biodyne, Tartu, Estonia), $0.7 \mu 1$ $50 \mathrm{mM} \mathrm{MgCl}_{2}$ (Solis Biodyne), $0.75 \mu 110 \mathrm{mM}$ deoxyribonucleotide triphosphates (Solis Biodyne), $2.5 \mu \mathrm{l} \mathrm{S}$ solution (Solis Biodyne), $0.3 \mu 1100 \mu \mathrm{M}$ forward and reverse primer (IDT, Coralville, IA, USA; primer sequences are listed in Table I) and $0.5 \mu \mathrm{l} 5 \mathrm{U} / \mu \mathrm{l}$ Hot Start DNA Polymerase (Solis Biodyne). Termocycling consisted in 1 cycle of enzyme activation (15 min at $95^{\circ} \mathrm{C}$ ) followed by 35 cycles of DNA amplification (45 sec at $95^{\circ} \mathrm{C}, 45 \mathrm{sec}$ at $59^{\circ} \mathrm{C}$ and $1 \mathrm{~min}$ at $72^{\circ} \mathrm{C}$ ). PCR products were then separated by agarose gel electrophoresis (1.5\% agarose gel in tris-borate-ethylenediaminetetraacetate; Sigma-Aldrich, St. Louis, MO, USA) and purified by Invisorb spin columns (Invitek, Hayward, CA, USA). PCR-purified products were re-amplified with terminated nuclotides employing Big Dye Terminator v3.1 (Applied Biosystems; Thermo Fisher Scientific, Waltham, MA, USA). Sequencing analysis was performed using an ABI Prism 3100 Avant automated sequencer (Thermo Fisher Scientific) equipped with $36-\mathrm{cm}$ capillary array filled with POP6 polymer (Thermo Fisher Scientific). Electropherograms were analyzed using Sequencing Analysis software (version 3.5; Applied Biosystems).

\section{Results}

Ophthalmological assessment. As illustrated in Fig. 1, the study included six members of an Italian family (four males and two females), among which three presented with OMD (subjects, II 1, III 1 and III 2).

The main patient (subject II 1) was a 67-year-old male. The BCVA was 'counting fingers' in both eyes (OU) without any

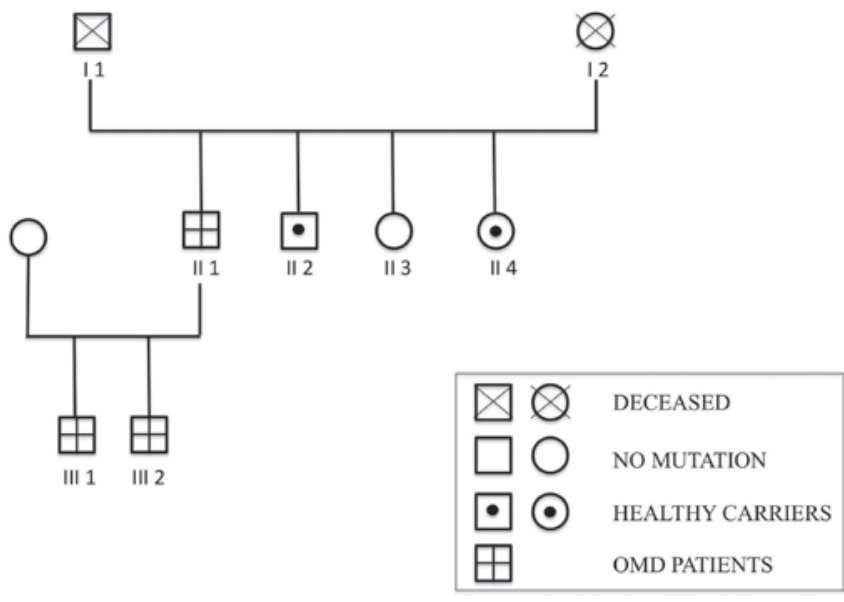

Figure 1. Pedigree of a caucasian family with OMD. The identification numbers of the study subjects are stated below the symbols. Circles, females; squares, males; OMD, occult macular dystrophy.

detectable alteration of the anterior and posterior segment of the eye. The patient's medical records revealed a progressive visual decline beginning at age 59. The results of the slit-lamp examination as well as intraocular pressure and fundus appearance were normal. The results of the color vision test was also normal until the BCVA reached 0.3 . Automated perimetry revealed a central relative scotoma in OU. Full-field ERG was within normal limits, whereas multi-focal ERG showed a reduction of the amplitudes in the central region. Clinical and instrumental findings were matched the diagnosis of OMD.

SD-OCT was then performed; macular scans showed that in the foveal region, the external limiting membrane (ELM) and the inner segment-outer segment (IS/OS) line were not sharply identifiable from each other. In addition, the cone outer segment tip (COST) line was disrupted (Fig. 2). A mild hyperreflectivity of the internal limiting membrane was also present, and central retinal thickness was reduced.

The second patient (subject III 1) was a 38-year-old male. The BCVA was 0.2 in OU, and no ocular abnormalities were detected by slit-lamp and fundus examination. The patient's medical records reported progressive visual decline, associ- 


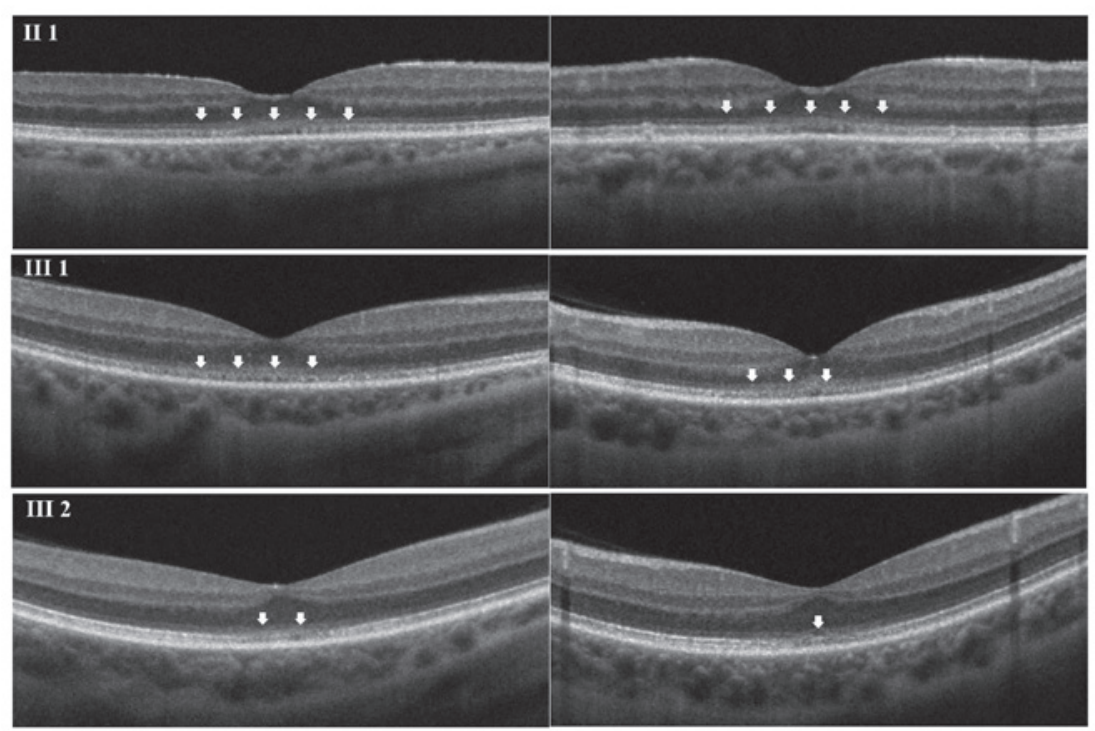

Figure 2. Spectral domain optical coherence tomography scans (left column, horizontal; right column, vertical) of right eye of subjects II 1 , III 1 and III 2 . In all three patients, the external limiting membrane and the inner segment/outer segment line appeared blurred, and the cone outer segment tip line was disrupted. Arrowheads indicate the areas affected by morphological changes to the outer retinal layers. The extent of macular alteration was correlated with the best-corrected visual acuity (counting fingers in subject II 1, 0.2 in subject III 1 and 0.6 in subject III 2).

ated with photophobia, starting at age 15 . Color vision testing showed a red-green defect and automated perimetry revealed a central relative scotoma in OU. SD-OCT results regarding the ELM, the IS/OS line and the COST line were similar to those observed in subject II 1; however, the affected area was smaller (Fig. 2).

Subject III 2 was a 32 year-old male. The subject's BCVA was 0.6 in OU, with no evidence of any changes in ocular morphology. Color vision testing showed normal results. The patient presented with a slow, progressive decline of visual acuity since age 28 . The SD-OCT findings were comparable to those in subjects II 1 and III 1, although the area involved was minimal (Fig. 2).

In addition, clinical assessment of three clinically asymptomatic siblings of subject II 1 (II 2, II 3 and II 4, a 51 year-old male, a 56 year-old female and a 66 year-old female, respectively) was performed (Fig. 1). All three subjects had a BCVA of 1.0 in $\mathrm{OU}$ and normal results of the ophthalmological examination. SD-OCT revealed that the retinal layers were normal.

Analysis of RPIL1 mutations. Subject II 1 was screened for the following targeted mutations in the RP1L1 gene: Ser1199Cys, Trp960Arg, Arg45Trp, Gln2311Pro, Gln2311Glu, Asp1425His and Asp1425Glu. Of these, the Arg45Trp mutation, which was reported by Akahori et al (8) to be associated with autosomal-dominant OMD, was identified in subject II (Fig. 3A). As Arg45Trp was previously found to be correlated with occult macular dystrophy, subjects III 1, III 2, II 2, II 3 and II 4 were also screened for this specific mutation. As expected due to their clinical diagnosis, subjects III 1 and III 2 were also carriers of the Arg45Trp mutation (Fig. 3B and C). While subjects II 2 and II 4 were also carriers of the Arg45Trp mutation, they displayed no clinical symptoms of OMD (Fig. 3D and F). The Arg45Trp mutation was not present in subject II3 (Fig. 3E). Therefore, in the study population, the Arg45Trp mutation was present in $3 / 5$ subjects $(60 \%)$.

\section{Discussion}

The prevalence of occult macular dystrophy (OMD) appears to be elevated Asian populations, as the majority of available studies have been performed in Japanese or Korean populations (1,4-10). Only two studies on Caucasian subjects with OMD are available, of which only one included an analysis for RP1L1 gene mutations $(11,12)$.

OMD is an autosomal inherited condition, although sporadic cases have also been reported. RP1L1 was identified as a gene responsible for the disease in 2010 (8). RP1L1 is located on chromosome $8 \mathrm{p}$ and originates from the common ancestor gene retinitis pigmentosa 1 (RP1) on chromosome 8 . RP1L1 shares $35 \%$ amino acid identity with RP1, a gene responsible for $51 \%$ of autosomal dominant retinitis pigmentosa cases worldwide $(8,11)$. RP1 is responsible for $5-10 \%$ of autosomal dominant retinitis pigmentosa cases worldwide (11). Although RP1L1 shares $35 \%$ amino acid identity with RP1, RP1L1 mutations are not associated with retinitis pigmentosa.

Immunohistochemical studies on RP1L1 in retinal sections of cynomolgus monkeys showed that the gene is expressed in rods as well as cones (8). Although the function of RP1L1 remains to be fully elucidated, it probably cooperates with RP1 to assemble and stabilize the microtubules of the photoreceptor axonemes (13).

While the link between OMD and RP1L1 mutations has been reported in Asian and north European pedigrees, the present study was the first to demonstrate that an RP1L1 mutation is likely to be associated with OMD in an Italian family. The substitution mutation p.Arg45Trp was identified in a patient who met the diagnostic criteria for OMD, as well as in the two symptomatic male offspring of the patient and also in two clinically asymptomatic siblings of the patient. Tsunoda et al (10), who reported on a Japanese family carrying the p.Arg45Trp mutation, found that the age of onset of OMD ranged from 6-50 years. It is often difficult to establish the true 

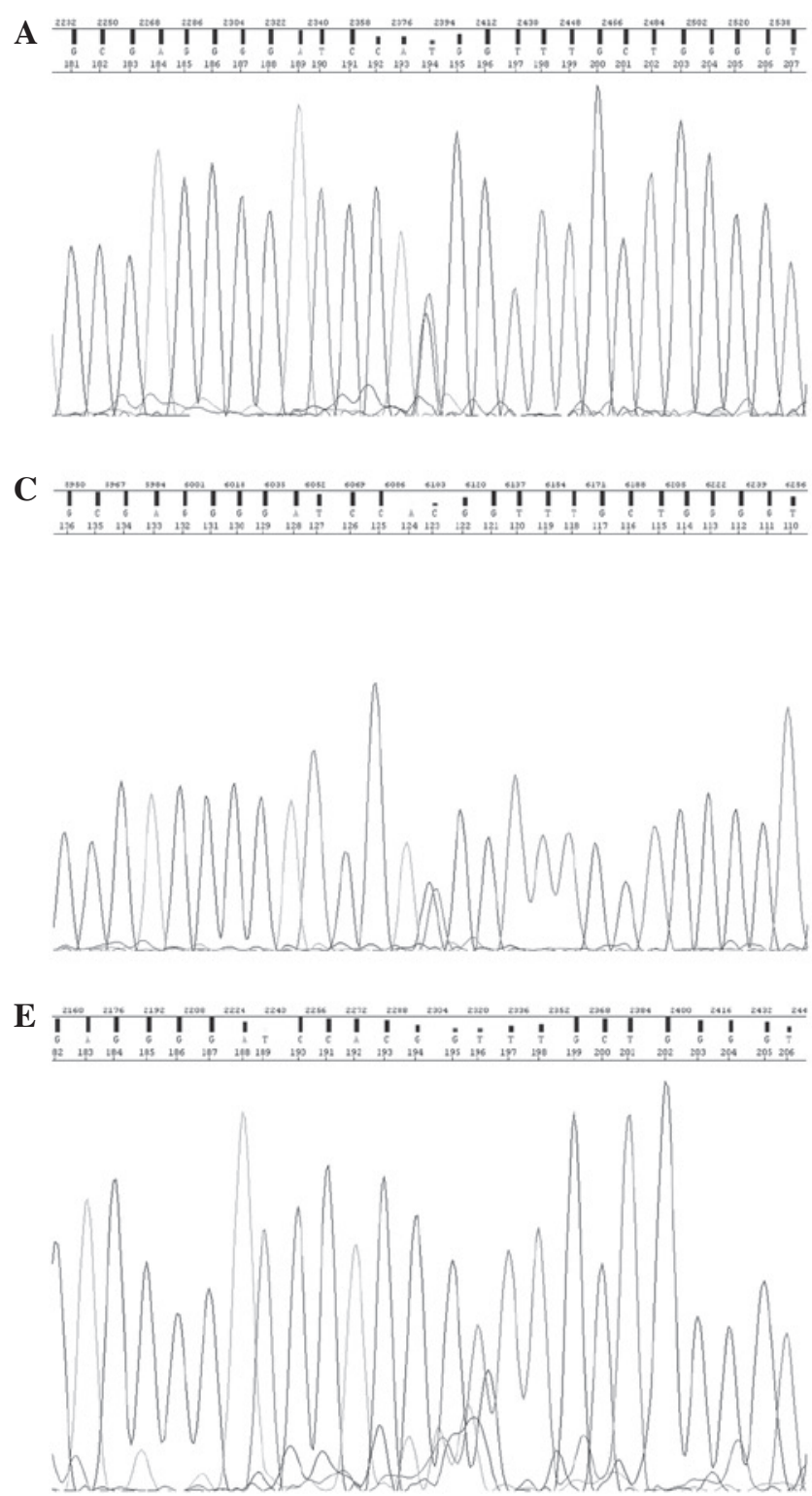

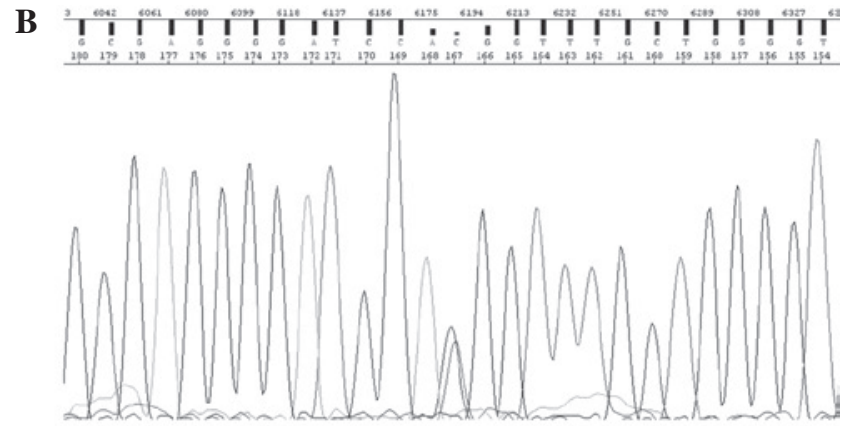

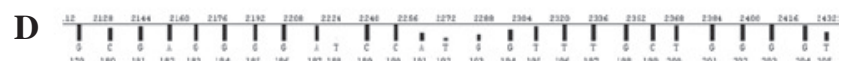

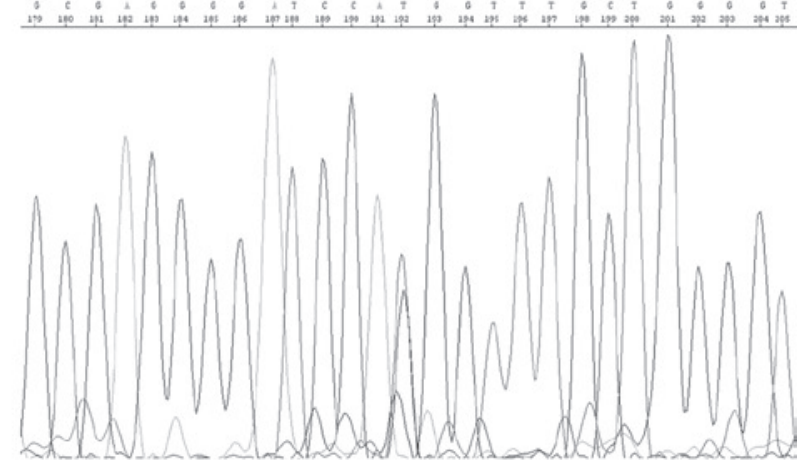

F

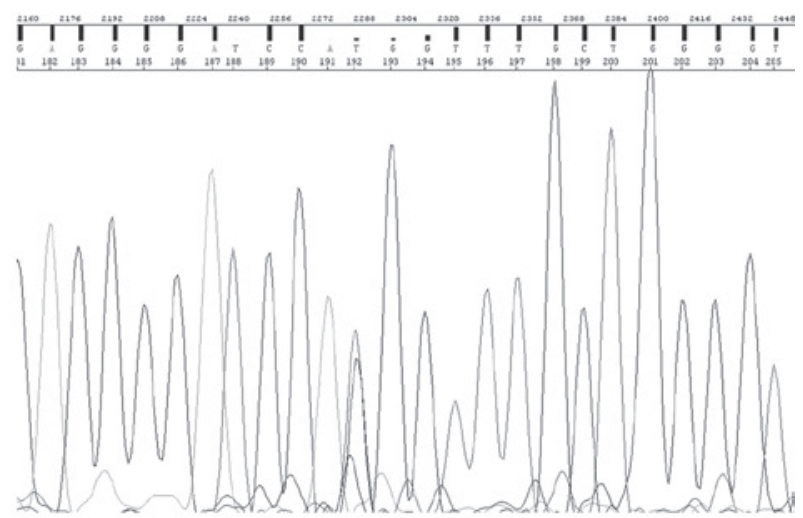

Figure 3. Arg45Trp electropherograms for the study subjects. (A) Subject II 1; (B) subject III 1; (C) subject III 2; (D) subject II 2; (E) subject II 4; (F) subject II 3.

age of onset of the disease, as certain patients with OMD have normal visual acuity and no subjective visual disturbance until the disease progresses to a more advanced stage (10). In the pedigree assessed in the present study, the age of first presentation of visual symptoms ranged from 15 years for subject III 1 to 59 years of age for subject II 1 . The onset of progressive BCVA reduction reported by Tsunoda et al (10) ranged from 6-50 years of age. Similarly, in the present study, the onset of progressive BCVA reduction varied. Similarly, in the present study, the rate of the progression of the visual decline varied. Subject II 1 became legally blind over a period of eight years, while subject III 1 showed a slower reduction of BCVA, which decreased to 0.2 over a period of 23 years. BCVA of the third proband decreased to 0.6 over three years; however, the time of observation was too short to predict the rate of progression. OMD has been reported to be a slowly progressing disease, while the visual function usually became stationary when the final visual acuity reached a value of 0.1-0.2 $(8,10)$. However, the BCVA of Subject II 1 deteriorated to counting fingers in OU.
The diagnosis of OMD is challenging, as no detectable fundus abnormalities are present at advanced stages. Despite normal fundus appearance, SD-OCT is able to identify a number of alterations in the outer retinal layers in the majority of OMD patients. Tomographic features of OMD include central retinal thinning, blurring of the ELM and IS/OS band as well as disruption of the COST line, whereas the RPE band is always preserved $(6-7,10)$. All of these characteristics were observed in the OMD patients of the present study. It has also been reported that the degree of these alterations worsens over time and correlates with visual impairment. In spite of the small number of patients included in the present study, it was indicated that a better visual acuity is correlated with less extensive macular alterations.

Thorough analysis of SD-OCT scans of asymptomatic subjects II 2, II 3 and II 4 revealed that no major or minimal alterations of the outer retinal layers were present. Tsunoda et al (10) reported that even though certain OMD patients showed a good BCVA or did not complain about any visual symptoms, the mfERG revealed the presence of a macular dysfunction. 
In conclusion, the present study was the first to demonstrate the occurrence of the p.Arg45Trp mutation in the RP1L1 gene in an Italian pedigree including OMD patients. The RP1L1 Arg45Trp mutation, at least in the cohort of the present study, appeared to have incomplete penetrance. Davidson et al (12) suggested the Arg45Trp mutation as a risk factor for OMD rather than a causative mutation. However, the reason for the incomplete penetrance displayed by individuals possessing the OMD-associated RP1L1 variant p.Arg45Trp remains elusive. A digenic or oligogenic model involving other genes requires further investigation. In the present study, the first of South-European origin, a disease penetrance of $60 \%$ was presented; this is higher than that observed by Davidson et al (12) (38\%), and more comparable with a study on Asian subjects by Akahori et al (8) (identified $85 \%$ penetrance). In addition Davidson et al (12) concluded that the Arg45Trp mutation is a risk factor, not a causative mutation, for OMD, based on the observation that none of their patients had familial history of autosomal-dominant maculopathy. In the current study, a Caucasian father and his two sons are described who presented with OMD, thus supporting the role of the Arg45TRp mutation in the cause of the disease in non-Asian subjects. Of note, as the age of onset for OMD is variable, clinical diagnosis of OMD is challenging and healthy carriers of the Arg45Trp mutation should be considered to be at risk, and periodic clinical screening for OMD is recommended.

Based on previous studies and the observations of the present study, it is indicated that screening for mutations in the RP1L1 gene associated with progressive reduction of BCVA and typical OCT per se may be sufficient to confirm the diagnosis of OMD. Under these circumstances, mfERG is not necessary (as in subjects III 1 and III 2). However, when visual symptoms or fundus abnormalities are absent, but genetic testing is positive, mfERG may be the only test with the capability of early detection of an impending macular dysfunction.

\section{References}

1. Miyake Y, Ichikawa K, Shiose Y and Kawase Y: Hereditary macular dystrophy without visible fundus abnormality. Am J Ophthalmol 108: 292-299, 1989.

2. Mattews GP, Sandberg MA and Berson EL: Foveal cone electroretinograms in patients with central visual loss of unexplained etiology. Arch Ophthalmol 110: 1568-1570, 1992.

3. Lyons JS: Non familial occult macular dystrophy. Doc Ophthalmol 111: 49-56, 2005.

4. Miyake Y, Horiguchi M, Tomita N, Kondo M, Tanikawa A, Takahashi H, Suzuki S and Terasaki H: Occult macular dystrophy. Am J Ophthalmol 122: 644-653, 1996.

5. Piao CH, Kondo M, Tanikawa A, Terasaki H and Miyake Y: Multifocal electroretinogram in occult macular dystrophy. Invest Ophthalmol Vis Sci 41: 513-517, 2000.

6. Park SJ, Woo SJ, Park KH, Hwang JM and Chung H: Morphologic photoreceptor abnormality in occult macular dystrophy on spectral-domain optical coherence tomography. Invest Ophthalmol Vis Sci 51: 3673-3679, 2010.

7. Ahn SJ, Ahn J, Park KH and Woo SJ: Multimodal imaging of occult macular dystrophy. JAMA Ophthalmol 131: 880-890, 2013.

8. Akahori M, Tsunoda K, Miyake Y, Fukuda Y, Ishiura H, Tsuji S, Usui T, Hatase T, Nakamura M, Ohde H, et al: Dominant mutations in RP1L1 are responsible for occult macular dystrophy. Am J Hum Genet 87: 424-429, 2010

9. Kabuto T, Takahashi H, Goto-Fukuura Y, Igarashi T, Akahori M, Kameya S, Iwata T, Mizota A, Yamaki K, Miyake Y and Takahashi H: A new mutation in RP1L1 gene in a patient with occult macular dystrophy associated with a depolarizing pattern of focal macular electroretinograms. Mol Vis 18: 1031-1039, 2012.

10. Tsunoda K, Usui T, Hatase T, Yamai S, Fujinami K, Hanazono G, Shinoda K, Ohde H, Akahori M, Iwata T and Miyake Y: Clinical characteristics of occult macular dystrophy in a family with mutation of RP1L1 gene. Retina 32: 1135-1147, 2012.

11. Jacobson SG, Cideciyan AV, Iannaccone A, Weleber RG, Fishman GA, Maguire AM, Affatigato LM, Bennett J, Pierce EA, Danciger M, et al: Disease expression of RP1 mutations causing autosomal dominant retinitis pigmentosa. Invest Ophthalmol Vis Sci 41: 1898-1908, 2000.

12. Davidson AE, Sergouniotis PI, Mackay DS, Wright GA, Waseem NH, Michaelides M, Holder GE, Robson AG, Moore AT, Plagnol V and Webster AR: RP1L1 variants are associated with a spectrum of inherited retinal diseases including retinitis pigmentosa and occult macular dystrophy. Hum Mutat 34: 506-514, 2013.

13. Yamashita T, Liu J, Gao J, LeNoue S, Wang C, Kaminoh J, Bowne SJ, Sullivan LS, Daiger SP, Zhang K, et al: Essential and synergistic roles of RP1 and RP1L1 in rod photoreceptor axoneme and retinitis pigmentosa. J Neurosci 29: 9748-9760, 2009. 